\title{
COMMUTATIVE SUBGROUPS AND TORSION IN COMPACT LIE GROUPS
}

\author{
BY ARMAND BOREL
}

Communicated by Deane Montgomery, April 26, 1960

In this note, $G$ is a compact connected Lie group. We are concerned with the torsion of the cohomology ring $H^{*}(G ; Z)$ of $G$ over the integers, certain commutative subgroups of $G$, and relations between these two questions.

Notation. $E\left(m_{1}, \cdots, m_{r}\right)$ or $E_{A}\left(m_{1}, \cdots, m_{r}\right)$ denotes the exterior algebra over the ring $A$ of a free $A$-module with $r$ generators of respective degrees $m_{1}, \cdots, m_{r} ; p$ is a prime number, $Z_{p}$ the field of integers $\bmod p, Q$ the field of rational numbers. Tors $H$ is the torsion subgroup of a group $H$, ord $M$ the order of a finite group $M$. The identity component of a closed subgroup $H$ if $G$ is denoted by $H_{0}$, if $L$ is a subset of $G$, its centralizer in $G$ is denoted by $Z(L)$.

1. $H$-spaces with finitely generated cohomology groups. In this section, $X$ is a compact connected $H$-space, for which $H^{*}(X ; Z)$ is finitely generated. As is known $H^{*}(X ; Q)=E\left(m_{1}, \cdots, m_{r}\right)$ with $m_{i}$ odd; we assume $m_{i} \leqq m_{j}$ if $i \leqq j$. With this notation we have

Proposition 1.1. (a) $H^{*}(X ; Z) /$ Tors $H^{*}(X ; Z)=E_{Z}\left(m_{1}, \cdots, m_{r}\right)$. (b) Let $p$ be odd and $K$ be a field of characteristic $p$. Then $H^{*}(X ; K)$ contains a subalgebra isomorphic to $E_{K}\left(m_{1}, \cdots, m_{r}\right)$. Each system of generators of type $(M)$ of $H^{*}(X ; K)$ (in the sense of $[2, \S 6]$ ) contains at least $r$ elements of odd degrees.

Let $H=H^{*}(X ; Z) /$ Tors $H^{*}(X ; Z)$. The transpose of the product map induces a homomorphism $H \rightarrow H \otimes H$ satisfying the conditions imposed on a Hopf algebra over $Z$. Hence $H \otimes A$ is a Hopf algebra over $A$ for any ring $A$. Then (a) follows from the structure theorem [2, Théorème 6.1] applied to $H \otimes L, L$ being a field, by an easy induction. (b) follows from (a), the structure theorem, and the fact that the product of the generators of $E_{K}\left(m_{1}, \cdots, m_{r}\right)$ is nonzero.

Proposition 1.2. Let $p$ be odd. A ssume that each element of $H^{*}\left(X ; K_{p}\right)$ has height $\leqq p$ (in the sense of $[2, \S 6]$ ) and that $X$ is simply connected. Let $k$ be the first integer such that $H^{k}(X ; Z)$ has p-torsion. Then $p \cdot k$ $\leqq m_{r}+p-1$.

This may be proved using the spectral sequence connecting $H^{*}\left(X ; Z_{p}\right)$ to $H^{*}(X ; Z) /$ Tors $H^{*}(X ; Z) \otimes Z_{p}$, whose differentials are the successive Bockstein operators. This result is sufficient for the 
applications mentioned in $\S 2$; however, since then, a more complete one has been obtained by Araki [1].

2. On the $p$-torsion of compact Lie groups. By $E_{i}(i=6,7,8)$ we mean here the compact simply connected group whose Lie algebra is denoted by that symbol in the standard Killing-Cartan classification. It was shown in [7] that $E_{i}$ has no $p$-torsion for $i=6, p \geqq 7$, and $i=7,8, p \geqq 11$. By Theorem V of [11], $E_{i}$ has the homology of the Eilenberg-MacLane space $K(Z, 3)$ up to dimension $m_{2}-1$ (in the notation of $\$ 1)$. Together with the results of $\mathrm{H}$. Cartan [12] on the homology of $K(\pi, n)$ this shows that $E_{6}, E_{7}, E_{8}$ have 2- and 3-torsion, and that $E_{8}$ also has 5-torsion. Combined with 1.1, 1.2, these results also allow one to prove the

Proposition 2.1. The group $E_{i}$ has no $p$-torsion for $i=6,7, p \geqq 5$ and for $i=8, p \geqq 7$. Furthermore

$$
\begin{aligned}
& H^{*}\left(E_{6} ; Z_{3}\right)=E(3,7,9,11,15,17) \otimes P(8 ; 3), \\
& H^{*}\left(E_{8} ; Z_{5}\right)=E(3,11,15,23,27,35,39,47) \otimes P(12 ; 5)
\end{aligned}
$$

where $P(a ; p)=Z_{p}[x] /\left(x^{p}\right)$, with $x$ of degree $a$.

Together with results of $[4 ; 7]$, this yields a verification of the second assertion conjectured in [6] of the following:

THEOREM 2.2. (a) $G$ has no p-torsion if and only if $B_{G}$ has no p-torsion. (b) Let $G$ be simple and simply connected. Then if $p$ does not divide the coefficients of the highest root, expressed as linear combination of the simple roots, then $G$ has no p-torsion.

For the "only if" part of (a) see [6, Proposition 5.1]. The "if" part is checked using 2.1 and [7]. The reduction to simple groups uses the fact that $G$ is homeomorphic to the product of $Z(G)_{0}$ by its greatest semi-simple subgroup. More generally, if $G=S \cdot G_{1}$ where $S$ is a torus and $G_{1}$ a closed connected subgroup, both invariant, then $G$ is homeomorphic to the product of a torus by $G_{1}$. Using induction on $\operatorname{dim} S$, it is enough to prove this when $S$ is a circle. Then, if $G \neq G_{1}$, the group $G$ is a principal $G_{1}$-bundle over a circle, and this bundle is trivial since $G_{1}$ is connected.

3. Commutative subgroups and $p$-torsion. As in [8], a [p]-group is a commutative group of type $(p, \cdots, p)$; its rank is the number of factors isomorphic to $Z_{p}$.

THEOREM 3.1. (a) Let $H$ be a compact commutative subgroup of $G$. 
Assume that $\operatorname{ord}\left(H / H_{0}\right)$ is prime to the order of Tors $\pi_{1}(G)$, and that either $H$ contains a regular element of $G$ or $H / H_{0}$ is generated by two elements. Then $H$ is contained in a torus of $G$. (b) $\pi_{1}(G)$ has no p-torsion if and only if every [p]-group of rank $\leqq 2$ belongs to a toral subgroup.

The proof of 3.1 uses notably the end remark of $\$ 2$, the fact that $G / Z(S)$ is torsion free when $S$ is a torus $[5 ; 10]$, and the fact that if $G$ is simply connected, the centralizer $Z(g)$ of any element of $g$ is connected, which can be proved using Proposition 2, p. 56 of [13] and some properties of singular elements. The assertion (b), in the direction not covered by (a), is checked by explicit construction of examples. The main property to verify is the following: Let $G$ be simple, simply connected, and $z$ be an element of order $p$ of the center of $G$. Then there exist $u, v \in G$ with $u v u^{-1} v^{-1}=z$ and $u^{p}=v^{p}=e$ if $p$ is odd, $u^{p}=v^{p}=z$ if $p=2 .{ }^{1}$

TheOREM 3.2. (a) Let $p$ be a prime. Then $G$ has no $p$-torsion if and only if every [p]-subgroup is contained in some torus. (b) Let $H$ be a compact commutative subgroup of $G$. Assume that ord $\left(H / H_{0}\right)$ is prime to the order of Tors $H^{*}(G, Z)$. Then $H$ is contained in a torus of $G$.

The "only if" part of (a) was proved in [3, Chapter XIII]. The "if" part is checked by explicit constructions. These show that if $G$ is simple, simply connected and has $p$-torsion, then $G$ contains a [p]-subgroup of rank 3 not belonging to a torus. The proof of (b) proceeds by induction on $\operatorname{dim} G$. It uses the following fact, which, when $U$ is not the centralizer of a torus, had to be checked using the classification of subgroups of maximal rank [9].

Proposition 3.3. Let $U$ be a connected closed subgroup of maximal rank of $G$. If $G$ has no p-torsion, then $U$ and $G / U$ have no p-torsion.

4. Remarks about maximal [2]-subgroups. It is known $[2 ; 3]$ that $H^{*}\left(G ; Z_{2}\right)$ has a simple system of universally transgressive generators when $G=S O(n), S p(n), S U(n),(n \geqq 1) G_{2}, F_{4}, \operatorname{Spin}(m)(m \leqq 9)$. In the first four cases, it was also shown [3] that maximal [2]subgroups are conjugate by inner automorphisms and play in cohomology mod 2 a role analogous to that of maximal tori in rational cohomology. This also holds in the remaining cases; more precisely:

THEOREM 4.1. The maximal [2]-subgroups of $G_{2}, F_{4}$, Spin (7), Spin (8), Spin (9) are conjugate by inner automorphisms, their ranks

1 Theorem 3.1(b) was suggested in discussions with J. Wolf, to whom I am also indebted for an example in the case of Spin $(4 n), p=2$. 
are equal to 3, 5, 4, 5, 5 respectively. If $U$ is one of them, then $G / U$ is totally nonhomologous to zero mod 2 in the fibering

$$
\left(B_{U}, B_{G}, G / U, \rho(U, G)\right) \text {. }
$$

\section{REFERENCES}

1. S. Araki, $A$ theorem of differential Hopf algebras and the cohomology mod 3 of the compact exceptional groups $E_{7}, E_{8}$, (to appear).

2. A. Borel, Sur la cohomologie des espaces fibrés principaux et des espaces homogènes de groupes de Lie compacts, Ann. of Math. vol. 57 (1953) pp. 115-207.

3. - La cohomologie modulo 2 de certains espaces homogènes, Comment. Math. Helv. vol. 27 (1953) pp. 165-197.

4. - Sur l'homologie et la cohomologie des groupes de Lie compacts connexes, Amer. J. Math. vol. 76 (1954) pp. 273-342.

5. - Kählerian coset spaces of semi-simple Lie groups, Proc. Nat. Acad. Sci. U.S.A. vol. 40 (1954) pp. 1147-1151.

6. - Topology of Lie groups and characteristic classes, Bull. Amer. Math. Soc. vol. 61 (1955) pp. 397-432.

7. - Sur la torsion des groupes de Lie, J. Math. Pures Appl. vol. 35 (1956) pp. $127-139$.

8. - Seminar on transformation groups, Annals of Mathematics Studies, no. 46, Princeton, 1960.

9. A. Borel and J. de Siebenthal, Les sous-groupes fermés connexes de rang maximum des groupes de Lie clos, Comment. Math. Helv. vol. 23 (1949-50) pp. 200-223.

10. R. Bott, An application of the Morse theory to the topology of Lie groups, Bull. Soc. Math. France vol. 84 (1956) pp. 251-281.

11. R. Bott and H. Samelson, Applications of the Morse theory to symmetric spaces, Amer. J. Math. vol. 80 (1958) pp. 964-1029.

12. H. Cartan, Séminaire E.N.S., 1954-1955 (mimeographed).

13. J. de Siebenthal, Sur les groupes de Lie compacts non connexes, Comment. Math. Helv. vol. 31 (1956) pp. 41-89.

INSTITUTE FOR ADVANCED STUdy 